\title{
ON RADIAL LIMIT FUNCTIONS FOR ENTIRE SOLUTIONS OF SECOND ORDER ELLIPTIC EQUATIONS IN $\mathbf{R}^{2}$
}

\author{
André Boivin* and Peter V. Paramonov ${ }^{\dagger}$
}

\begin{abstract}
Given a homogeneous elliptic partial differential operator $L$ of order two with constant complex coefficients in $\mathbf{R}^{2}$, we consider entire solutions of the equation $L u=0$ for which

$$
\lim _{r \rightarrow \infty} u\left(r e^{i \varphi}\right)=: U\left(e^{i \varphi}\right)
$$

exists for all $\varphi \in[0,2 \pi)$ as a finite limit in $\mathbf{C}$. We characterize the possible "radial limit functions" $U$. This is an analog of the work of A. Roth for entire holomorphic functions. The results seem new even for harmonic functions.
\end{abstract}

\section{Introduction and Main Results}

Let

$$
L v=c_{11} v_{x_{1} x_{1}}+2 c_{12} v_{x_{1} x_{2}}+c_{22} v_{x_{2} x_{2}}
$$

be an homogeneous partial differential operator of order two with constant complex coefficients in $\mathbf{R}^{2}$ satisfying the ellipticity condition

$$
c_{11} \xi_{1}^{2}+2 c_{12} \xi_{1} \xi_{2}+c_{22} \xi_{2}^{2} \neq 0
$$

for all $\left(\xi_{1}, \xi_{2}\right) \neq(0,0), \xi_{1}, \xi_{2} \in \mathbf{R}$.

Keywords. Elliptic operator, $L$-entire functions, radial limit functions.

* The first author was partially supported by NSERC (Canada).

†The second author was supported by RFBR (grants No 96-01-01240 \& 96-15-96846). 
Let $\lambda_{1}, \lambda_{2}$ be the (complex) roots of the characteristic equation $c_{11} \lambda^{2}+$ $2 c_{12} \lambda+c_{22}=0$. It follows from the ellipticity condition that $\lambda_{1}, \lambda_{2} \notin \mathbf{R}$. We define

$$
\partial_{1}=\frac{\partial}{\partial x_{1}}-\lambda_{1} \frac{\partial}{\partial x_{2}}, \quad \partial_{2}=\frac{\partial}{\partial x_{1}}-\lambda_{2} \frac{\partial}{\partial x_{2}} \quad \text { if } \lambda_{1} \neq \lambda_{2},
$$

or

$$
\partial_{1}=\frac{\partial}{\partial x_{1}}-\lambda_{1} \frac{\partial}{\partial x_{2}}, \quad \partial_{2}=\frac{\partial}{\partial x_{1}}+\lambda_{1} \frac{\partial}{\partial x_{2}} \quad \text { if } \lambda_{1}=\lambda_{2} .
$$

We then have the following decomposition of $L$ :

$$
L v= \begin{cases}c_{11} \partial_{1}\left(\partial_{2}(v)\right), & \text { if } \lambda_{1} \neq \lambda_{2} ; \\ c_{11} \partial_{1}^{2}(v), & \text { if } \lambda_{1}=\lambda_{2} .\end{cases}
$$

We also introduce the following new coordinates:

$$
z_{1}=\frac{\lambda_{2}}{\lambda_{2}-\lambda_{1}}\left(x_{1}+\frac{1}{\lambda_{2}} x_{2}\right), \quad z_{2}=\frac{\lambda_{1}}{\lambda_{1}-\lambda_{2}}\left(x_{1}+\frac{1}{\lambda_{1}} x_{2}\right) \quad \text { if } \lambda_{1} \neq \lambda_{2} ;
$$

or

$$
z_{1}=\frac{1}{2}\left(x_{1}-\frac{1}{\lambda_{1}} x_{2}\right), \quad z_{2}=\frac{1}{2}\left(x_{1}+\frac{1}{\lambda_{1}} x_{2}\right) \quad \text { if } \lambda_{1}=\lambda_{2} .
$$

The following "orthogonality" relations then are easily obtained:

$$
\begin{array}{ll}
\partial_{1} z_{1}=1 & \partial_{1} z_{2}=0 \\
\partial_{2} z_{1}=0 & \partial_{2} z_{2}=1
\end{array}
$$

Finally, we identify $z=x_{1}+i x_{2}$ in $\mathbf{C}$ and $x=\left(x_{1}, x_{2}\right)$ in $\mathbf{R}^{2}$ and, for $s=1$ and 2, we define $T_{s}(z)=z_{s}$ (which are linear nondegenerate transformations of $\mathbf{R}^{2}$ ).

For any set $E$ in $\mathbf{R}^{2}$, denote by $L(E)$ the family of all functions $v$, each defined on its own neighbourhood $\Omega_{v}$ of $E$, such that $L v=0$ in $\Omega_{v}$ in the classical sense. We note that for $E$ open, one can take $\Omega_{v}=E$ for all $v$. Functions in $L(E)$ and $L\left(\mathbf{R}^{2}\right)$ are called $L$-analytic on $E$ and $L$-entire respectively.

It is well known that (for $E$ open) each function $v \in L(E)$ is realanalytic on $E$, and that each continuous function $v$ satisfying $L v=0$ on $E$ in the distributional sense is in $L(E)$. From these facts, using (1), one can prove the following well known result [1, Chapter IV, §6, (4.77)] (see also [5] for a simple direct proof). 
Proposition 1. Let $D$ be any domain in $\mathbf{C}$ and $L$ be as above.

1. If $D$ is simply connected and if $\lambda_{1} \neq \lambda_{2}$, then

1a) $v \in L(D)$ if and only if there exist $f_{1}$ holomorphic in $T_{1}(D)$ and $f_{2}$ holomorphic in $T_{2}(D)$ such that

$$
v(z)=f_{1}\left(T_{1}(z)\right)+f_{2}\left(T_{2}(z)\right)=f_{1}\left(z_{1}\right)+f_{2}\left(z_{2}\right)
$$

for all $z \in D$. In particular, L-entire functions $u$ are of the form $u(z)=f_{1}\left(z_{1}\right)+f_{2}\left(z_{2}\right)$ where $f_{1}, f_{2}$ are entire holomorphic functions.

1b) There exist in $\mathbf{C} \backslash\{0\}$ a fixed analytic branch $\log \left(z_{1} z_{2}^{\nu}\right)$ of the multivalued function $\log \left(z_{1} z_{2}^{\nu}\right)$ and a nonzero complex constant $C_{L}$ depending only on $L$ such that

$$
\Phi_{L}(z)=C_{L} \log \left(z_{1} z_{2}^{\nu}\right)
$$

is a fundamental solution of $L$, where $\nu=1$ if $\operatorname{sgn}\left(\operatorname{Im} \lambda_{1}\right) \neq$ $\operatorname{sgn}\left(\operatorname{Im} \lambda_{2}\right)$, and $\nu=-1$ otherwise.

2. If $\lambda_{1}=\lambda_{2}$, then

2a) $v \in L(D)$ if and only if there exist $g_{1}$ and $g_{2}$ holomorphic in $T_{2}(D)$ such that

$v(z)=T_{1}(z) g_{1}\left(T_{2}(z)\right)+g_{2}\left(T_{2}(z)\right)=z_{1} g_{1}\left(z_{2}\right)+g_{2}\left(z_{2}\right)$

for all $z \in D$. In particular, L-entire functions $u$ are of the form $u(z)=z_{1} g_{1}\left(z_{2}\right)+g_{2}\left(z_{2}\right)$ where $g_{1}, g_{2}$ are entire holomorphic functions.

2b) $\Phi_{L}(z)=C_{L} \frac{z_{1}}{z_{2}}$ is a fundamental solution of $L$, where $C_{L}$ is a nonzero complex constant depending only on $L$.

3. If $\left\{v_{n}\right\} \subset L(D)$ and $\left\{v_{n}\right\}$ converges uniformly to $v$ on compact subsets of $D$ as $n \longrightarrow \infty$, then $v \in L(D)$.

We just note that $1 \mathrm{~b}$ ) and $2 \mathrm{~b}$ ) follow from 1a) and 2a) respectively, and from the definition of fundamental solution. It is not difficult to check that if $\operatorname{sgn}\left(\operatorname{Im} \lambda_{1}\right) \neq \operatorname{sgn}\left(\operatorname{Im} \lambda_{2}\right)$ (respectively $\left.\operatorname{sgn}\left(\operatorname{Im} \lambda_{1}\right)=\operatorname{sgn}\left(\operatorname{Im} \lambda_{2}\right)\right)$, then the increment of the polar argument of $\left(z_{1} z_{2}\right)$ (respectively $\left(z_{1} / z_{2}\right)$ ) around the origin is zero, and thus some analytic branch of the function $\log \left(z_{1} z_{2}\right)$ (respectively $\log \left(z_{1} / z_{2}\right)$ ) exists in $\mathbf{R}^{2} \backslash\{(0,0)\}$. 
Example 1. For the Laplacian $L=\Delta$, one has $\lambda_{1}=i, \lambda_{2}=-i$, $z_{1}=z / 2, z_{2}=\bar{z} / 2$ and

$$
\begin{aligned}
& \partial_{1}=\frac{\partial}{\partial x_{1}}-i \frac{\partial}{\partial x_{2}}=: 2 \frac{\partial}{\partial z} \\
& \partial_{2}=\frac{\partial}{\partial x_{1}}+i \frac{\partial}{\partial x_{2}}=: 2 \frac{\partial}{\partial \bar{z}} \\
& \Phi_{\Delta}(z)=\frac{1}{4 \pi} \log \left(\frac{z \bar{z}}{4}\right) .
\end{aligned}
$$

For the Bitsadze operator

$$
L=\frac{\partial^{2}}{\partial \bar{z}^{2}}=\frac{1}{4}\left(\frac{\partial^{2}}{\partial x_{1}^{2}}+2 i \frac{\partial^{2}}{\partial x_{1} \partial x_{2}}-\frac{\partial^{2}}{\partial x_{2}^{2}}\right),
$$

one gets $\lambda_{1}=\lambda_{2}=-i, z_{1}=\bar{z} / 2, z_{2}=z / 2$ and

$$
\partial_{1}=2 \frac{\partial}{\partial \bar{z}}, \quad \partial_{2}=2 \frac{\partial}{\partial z}, \quad \Phi_{L}(z)=\frac{1}{\pi} \frac{\bar{z}}{z} .
$$

In order to formulate our main results (Theorems 1 and 2), we need the following characterization of radially constant solutions of the equation $L v=0$.

Proposition 2. Let $J=\left\{z \in \mathbf{C}: \varphi_{1}<\arg z<\varphi_{2}\right\}, \varphi_{1}<\varphi_{2} \leq$ $\varphi_{1}+2 \pi$ denote an (infinite) open sector with vertex at 0 . Let $v \in L(J)$ and assume that $v(z)=v\left(r e^{i \varphi}\right)=v\left(e^{i \varphi}\right)$ does not depend on $r$.

1. If $\lambda_{1} \neq \lambda_{2}$, then there exist $\alpha, \beta \in \mathbf{C}$ and a fixed analytic branch $\log \left(z_{1} / z_{2}\right)$ of $\log \left(z_{1} / z_{2}\right)$ in $J$ such that, for $z \in J$,

$$
v(z)=\alpha \log \frac{z_{1}}{z_{2}}+\beta
$$

$(2)$

$$
=\alpha \log \left(\frac{\cos \varphi+\frac{1}{\lambda_{2}} \sin \varphi}{\cos \varphi+\frac{1}{\lambda_{1}} \sin \varphi}\right)+\beta=: v_{12}^{*}\left(e^{i \varphi}\right) \text {. }
$$

2. If $\lambda_{1}=\lambda_{2}$, then there exist $\alpha, \beta \in \mathbf{C}$ such that, for $z \in J$,

$$
\begin{aligned}
v(z) & =\alpha \frac{z_{1}}{z_{2}}+\beta \\
& =\alpha\left(\frac{\cos \varphi-\frac{1}{\lambda_{1}} \sin \varphi}{\cos \varphi+\frac{1}{\lambda_{1}} \sin \varphi}\right)+\beta=: v_{1}^{*}\left(e^{i \varphi}\right) .
\end{aligned}
$$

(For this case, $J=\mathbf{C} \backslash\{0\}$ is also allowed.) 
Example 2. For $L=\Delta$, one has $v_{12}^{*}\left(e^{i \varphi}\right)=\alpha \varphi+\beta, \varphi_{1}<\varphi<\varphi_{2}$, and for $L=\partial^{2} / \partial \bar{z}^{2}, v_{1}\left(e^{i \varphi}\right)=\alpha e^{-2 i \varphi}+\beta$, where $\alpha$ and $\beta$ are any complex constants.

Theorem 1. Let $u$ be an entire solution of the equation $L u=0$ such that

$$
\lim _{r \rightarrow+\infty} u\left(r e^{i \varphi}\right)=: U\left(e^{i \varphi}\right)
$$

exists and is finite for all $\varphi \in[0,2 \pi)$. Then

A) $U$ is of Baire class 1 on $S=\left\{e^{i \varphi}: \varphi \in[0,2 \pi)\right\}$; that is, $U$ is a pointwise limit on $S$ of a sequence of continuous functions on $S$.

B) There is an open set $I=\cup_{j=1}^{\infty} I_{j}$, where the $I_{j}$ are disjoint open arcs on $S$ (and $I_{j}=\emptyset$ is possible for some $j$, but $I_{j} \neq S$ ) with the following properties:

B1) I is everywhere dense on $S$;

B2) On each $I_{j}, U\left(e^{i \varphi}\right)$ is of the form $v_{12}^{*}\left(e^{i \varphi}\right)$ if $\lambda_{1} \neq \lambda_{2}$ (respectively of the form $v_{1}^{*}\left(e^{i \varphi}\right)$, if $\left.\lambda_{1}=\lambda_{2}\right)$, (see (2) and (3));

B3) The limit (4) is uniform on each compact subset of each $I_{j}$.

Conversely, let $U$ be a function defined on $S$ and $I$ be an open subset of $S$ with $I=\cup_{j=1}^{\infty} I_{j}$, where the $I_{j}$ are disjoint open arcs. If $(A),(B 1)$ and (B2) above are satisfied, then there exists an L-entire function $u$ with the properties:

a) $\lim _{r \rightarrow \infty} u\left(r e^{i \varphi}\right)=U\left(e^{i \varphi}\right)$ for each $\varphi$;

b) The limit in (a) holds uniformly on each compact subset of $I_{j}$ for each $j$.

Moreover, if $U_{1}$ is of Baire class 1 on $S$ and $U_{1}\left(e^{i \varphi}\right)=\partial U\left(e^{i \varphi}\right) / \partial \varphi$ on $I$, then the function $u$ can be chosen such that (a) and (b) are satisfied and

$$
\lim _{r \rightarrow+\infty} \frac{\partial u\left(r e^{i \varphi}\right)}{\partial r}=0, \quad \lim _{r \rightarrow+\infty} \frac{\partial u\left(r e^{i \varphi}\right)}{\partial \varphi}=U_{1}\left(e^{i \varphi}\right)
$$

for all $\varphi \in[0,2 \pi)$.

Let $K$ be a compact set in $S$. Let $R P(K)$ (respectively $R U(K)$ ) denote the set of all functions $g$ on $K$ for which there exists $u=u_{g} \in L\left(\mathbf{R}^{2}\right)$ such that $u\left(r e^{i \varphi}\right) \longrightarrow g\left(e^{i \varphi}\right)$ for each $\varphi \in K$ (respectively $u\left(r e^{i \varphi}\right) \longrightarrow g\left(e^{i \varphi}\right)$ uniformly on $K$ ) as $r \rightarrow \infty$. 


\section{Theorem 2.}

a) For each compact set $K$ in $S, g \in R P(K)$ if and only if $g$ is of Baire class 1 on $K$ and there exists a countable family of disjoint open arcs $\left\{I_{j}\right\}_{j=1}^{\infty}$ in $K$ such that $K \backslash \cup_{j=1}^{\infty} I_{j}$ is nowhere dense in $S$ and on each $I_{j}, g$ is of the form $v_{12}^{*}\left(e^{i \varphi}\right)$ (when $\left.\lambda_{1} \neq \lambda_{2}\right)$ or $v_{1}^{*}\left(e^{i \varphi}\right)$ (when $\lambda_{1}=\lambda_{2}$ ) (see Proposition 2). In particular, $R P(K)$ consists of all Baire class 1 functions on $K$ if and only if $K$ has an empty interior on $S$.

b) Let $K$ be a compact set in $S, K \neq S$. Then $g \in R U(K)$ if and only if $g \in C(K)$ and $g$ is of the form $v_{12}^{*}\left(e^{i \varphi}\right)$ (when $\lambda_{1} \neq \lambda_{2}$ ) or $v_{1}^{*}\left(e^{i \varphi}\right)$ (when $\lambda_{1}=\lambda_{2}$ ) in each connected component of the interior of $K$ in $S$. In particular, $R U(K)=C(K)$ if and only if $K$ is nowhere dense in $S$. If $K=S$, then $R U(K)$ contains only constant functions.

\section{Proofs}

We first establish the following uniqueness theorem for $L$-analytic functions.

Lemma 1. Let $D$ be any domain in $\mathbf{C}$ and $v \in L(D)$. If the set $G_{v}=\left\{z=x_{1}+i x_{2} \in D \mid \nabla v(z):=\left(\partial v(z) / \partial x_{1}, \partial v(z) / \partial x_{2}\right)=(0,0)\right\}$ has at least one accumulation point inside $D$, then $v$ is constant in $D$.

Proof: From Proposition 1 and equations (1), one has $\partial_{1} v=f_{1}^{\prime}\left(z_{1}\right)$ for $\lambda_{1} \neq \lambda_{2}$ and $\partial_{1} v=g_{1}\left(z_{2}\right)$ for $\lambda_{1}=\lambda_{2}$, where $f_{1}^{\prime}$ and $g_{1}$ are holomorphic on $T_{1}(D)$ and $T_{2}(D)$ respectively. By assumption, $f_{1}^{\prime}=0$ on $T_{1}\left(G_{v}\right)$ (respectively $g_{1}=0$ on $T_{2}\left(G_{v}\right)$ ). It thus follows from the uniqueness theorem for holomorphic functions that $f_{1} \equiv$ const in $T_{1}(D)$ (respectively $g_{1} \equiv 0$ in $\left.T_{2}(D)\right)$. An analogous study of $\partial_{2} v$ completes the proof of Lemma 1.

Proof of Proposition 2: We shall consider only the case $\lambda_{1} \neq \lambda_{2}$, the proof for the case $\lambda_{1}=\lambda_{2}$ being similar. Let $v \in L(J), v=v\left(e^{i \varphi}\right)$. Let $v_{0}(z)=\log \left(z_{1} / z_{2}\right)$ be some fixed analytic branch of $\log \left(z_{1} / z_{2}\right)$ in $J$. Simple calculations show that $\partial v_{0}(z) / \partial \varphi \neq 0$ and $\partial v_{0} / \partial r \equiv 0$ in $J$. Fixing some $\varphi_{0} \in\left(\varphi_{1}, \varphi_{2}\right)$, we can thus find $\alpha$ and $\beta$ in $\mathbf{C}$ such that $v-\alpha v_{0}-\beta=0$ and $\partial\left(v-\alpha v_{0}-\beta\right) / \partial \varphi=0$ on the ray $\left\{\arg z=\varphi_{0}\right\}$. It thus follows that $\nabla\left(v-\alpha v_{0}-\beta\right)=0$ on the ray $\left\{\arg z=\varphi_{0}\right\}$. Lemma 1 now gives the desired result. 
Proof of Theorem 1: The scheme of the proof is analogous to that of A. Roth [7] (see also [3, Chapter IV, $\S 5 \mathrm{~A}]$ ). The main new tools are some recent results in approximation theory $([\mathbf{6}]$ and $[\mathbf{2}])$.

Let $u \in L\left(\mathbf{R}^{2}\right)$ satisfy (4), then $\left.\mathrm{A}\right)$ is a consequence of $\lim _{n \rightarrow \infty} u\left(n e^{i \varphi}\right)=$ $U\left(e^{i \varphi}\right)$. Using a decreasing sequence of nested intervals and condition (4), one can prove that for each nonempty sector $J^{\prime \prime}$ with vertex at the origin, there exists a nonempty sector $J^{\prime}=\left\{\varphi_{1}^{\prime}<\arg z<\varphi_{2}^{\prime}\right\} \subset J^{\prime \prime}$ with $\varphi_{1}^{\prime}<\varphi_{2}^{\prime} \leq \varphi_{1}^{\prime}+2 \pi$ such that $u$ is bounded on $J^{\prime}$ (see [3, p. 164]). Fix any $\varphi_{1}$ and $\varphi_{2}$ with $\varphi_{1}<\varphi_{2}$ and $\left[\varphi_{1}, \varphi_{2}\right] \subset\left(\varphi_{1}^{\prime}, \varphi_{2}^{\prime}\right)$. Let $u_{n}(z)=u\left(2^{n} z\right)$. We claim that the sequence $\left\{u_{n}(z)\right\}_{n=1}^{\infty}$ converges uniformly on compact subsets of the "closed" sector $J=\left\{\varphi_{1} \leq \arg z \leq \varphi_{2}\right\}$. From (4), it will follow that the limit function $v$ does not depend on $r$. Since $v \in L(J)$ (see 3 of Proposition 1), Proposition 2 will give us B) in our theorem (see [3, p. 166] for more details). To prove the claim, it suffices to establish that $\left\{u_{n}\right\}$ converges uniformly on the compact set $K=\left\{\varphi_{1} \leq\right.$ $\left.\arg z \leq \varphi_{2}, 1 \leq|z| \leq 2\right\}$. In order to prove this last assertion, it is enough to check that $\left|\nabla u_{n}\right|$ is uniformly bounded on $K$ and to use AscoliArzela's theorem. Notice that $\sup \left\{\left|u_{n}(z)\right| \mid z \in J^{\prime}, n \geq 1\right\}<+\infty$, and $d:=\operatorname{dist}\left(K, \partial J^{\prime}\right)>0$ (here and in the sequel, $\partial E$ is the boundary of a set $E)$. Denote by $\Phi$ the fundamental solution of $L$, which is found in Proposition 1 , and set $B(a, \delta)=\{z \in \mathbf{C}|| z-a \mid<\delta\}$, where $a \in \mathbf{C}$ and $\delta>0$. Fix $\psi \in C_{0}^{\infty}(B(0, d))$ such that $\psi=1$ in $B(0, d / 2)$. Now fix $z_{0} \in K$ and put $\psi_{0}(z)=\psi\left(z-z_{0}\right)$. Then $\psi_{0}=0$ outside the ball $B\left(z_{0}, d\right) \subset J^{\prime}$ and $\psi=1$ on $B\left(z_{0}, d / 2\right)$. One has $\left(\left[6\right.\right.$, p. 255]) $u_{n} \psi=\Phi * L\left(u_{n} \psi\right)$, so that in $B\left(z_{0}, d / 2\right)$, we can write (in the case $\lambda_{1} \neq \lambda_{2}$ )

$$
u_{n}(z)=\Phi *\left(L u_{n} \psi+a_{11} \partial_{1} u_{n} \partial_{2} \psi+a_{11} \partial_{2} u_{n} \partial_{1} \psi+u_{n} L \psi\right)(z)
$$

Since $\psi L u_{n} \equiv 0$ and $a_{11} \partial_{s} u_{n} \partial_{3-s} \psi=a_{11} \partial_{s}\left(u_{n} \partial_{3-s} \psi\right)-u_{n} L \psi(s=1$ and 2), we obtain that, in $B\left(z_{0}, d / 2\right)$,

$$
\begin{aligned}
u_{n} & =\Phi *\left(a_{11} \partial_{1}\left(u_{n} \partial_{2} \psi\right)+a_{11} \partial_{2}\left(u_{n} \partial_{1} \psi\right)-u_{n} L \psi\right) \\
& =a_{11}\left(\partial_{1} \Phi\right) *\left(u_{n} \partial_{2} \psi\right)+a_{11}\left(\partial_{2} \Phi\right) *\left(u_{n} \partial_{1} \psi\right)-\Phi *\left(u_{n} L \psi\right) .
\end{aligned}
$$

Now the desired uniform estimate for $\left|\nabla u_{n}\left(z_{0}\right)\right|$ can be obtained by making trivial estimates in the formula

$$
\begin{aligned}
\nabla u_{n}\left(z_{0}\right)=a_{11}\left[\left(\nabla \partial_{1} \Phi\right) *\left(u_{n} \partial_{2} \psi\right)+\left(\nabla \partial_{2} \psi\right)\right. & \left.*\left(u_{n} \partial_{1} \psi\right)\right] \\
& \left.-(\nabla \Phi) *\left(u_{n} L \psi\right)\right)\left.\right|_{z=z_{0}}
\end{aligned}
$$

The proof for the case $\lambda_{1}=\lambda_{2}$ is similar. 
Let us now prove the second part of Theorem 1. Let $I=\cup_{j=1}^{\infty} I_{j}, U$, $U_{1}$ be as in (the second part of) Theorem 1. Put $I_{0}=S \backslash I$, and for $j=0,1, \ldots$ let $J_{j}=\left\{z \in \mathbf{C} \backslash\{0\} \mid e^{i \arg (z)} \in I_{j}\right\}$. Finally set $F_{0}=$ $\left\{z \in J_{0}|| z \mid \geq 1\right\}, F_{j}=\left\{z \in J_{j} \mid \operatorname{dist}\left(z, \partial J_{j}\right) \geq 1\right\}, j=1,2, \ldots$, and $F=\cup_{j=0}^{\infty} F_{j}$. Notice that each $F_{j}$ and $F$ are closed subsets of $\mathbf{C}$ and that the $F_{j}(j \geq 0)$ are pairwise disjoint. We note that if they are infinitely many $F_{j}$, they are pushed to $\infty$ (i.e. they are eventually outside any fixed compact set). It follows that there exist pairwise disjoint neighbourhoods $\Omega_{j}$ of $F_{j}, j=0,1, \ldots$, with $\Omega_{j} \subset J_{j}$ for $j \geq 1$.

We first want to show that there exists a neighbourhood $\Omega_{0}^{\prime}$ of $F_{0}$, $\Omega_{0}^{\prime} \subset \Omega_{0}$, and a function $f \in C_{\mathrm{loc}}^{1}\left(\Omega_{0}^{\prime}\right)$ such that

$$
\begin{aligned}
& \lim _{r \rightarrow \infty} f\left(r e^{i \varphi}\right)=U\left(e^{i \varphi}\right), \\
& \lim _{r \rightarrow \infty} \frac{\partial f\left(r e^{i \varphi}\right)}{\partial \varphi}=U_{1}\left(e^{i \varphi}\right), \\
& \lim _{r \rightarrow \infty} \frac{\partial f\left(r e^{i \varphi}\right)}{\partial r}=0,
\end{aligned}
$$

for each $e^{i \varphi} \in I_{0}$. The proof of this elementary fact is included for completeness.

Let $\left.A_{0}=\{|z|<2\}, A_{s}=\left\{2^{s-1}<|z|<2^{s+1}\right\}\right), s=1,2, \ldots$, and let $\left\{\chi_{s}\right\}_{s=0}^{\infty}$ be a partition of unity on $\mathbf{C}$ subordinate to $\left\{A_{s}\right\}_{s=0}^{\infty}$ such that $\chi_{s}(z)=\chi_{s}(|z|)$ and $\left|\nabla \chi_{s}\right| \leq c / 2^{s}$, where $c$ is a constant independent of $s$. Since $U$ and $U_{1}$ are of Baire class 1 on $S$, there exist sequences of continuous functions $\left\{V_{s}\right\},\left\{W_{s}\right\}$ on $S$ such that $V_{s}\left(e^{i \varphi}\right) \longrightarrow U\left(e^{i \varphi}\right)$ and $W_{s}\left(e^{i \varphi}\right) \longrightarrow U_{1}\left(e^{i \varphi}\right)$, for all $e^{i \varphi} \in S$ (and thus in particular for all $e^{i \varphi} \in I_{0}$ ). In addition we can choose the continuous functions $V_{s}$ and $W_{s}$ so that they are bounded by $2^{s / 2}$.

Since $V_{s}$ and $W_{s}$ are uniformly continuous on $S$, there exists $\delta_{s}, 0<$ $\delta_{s}<2^{-s}$, such that $\left|e^{i \varphi}-e^{i \varphi_{0}}\right|<\delta_{s}$ implies $\left|V_{s}\left(e^{i \varphi}\right)-V_{s}\left(e^{i \varphi_{0}}\right)\right|<1 / 2^{s}$ and $\left|W_{s}\left(e^{i \varphi}\right)-W_{s}\left(e^{i \varphi_{0}}\right)\right|<1 / 2^{s}$.

Since by assumption $I_{0}$ is nowhere dense in $S$, there exist open neighbourhoods $N_{s}$ of $I_{0}, s=0,1, \ldots$, such that $N_{s}=\cup_{k \geq 1} I_{s k}$ is the union of finitely many open $\operatorname{arcs} I_{s k}$ whose closures are disjoint and each $I_{s k}$ is of length less than $\delta_{s}$.

Now for each $s \geq 0$, define $\Omega_{0}^{s}=N_{s}^{(\varphi)} \times\left(2^{s-1}, 2^{s+1}\right)^{(r)}$ and $\Omega_{0}^{s k}=$ $I_{s k}^{(\varphi)} \times\left(2^{s-1}, 2^{s+1}\right)^{(r)}$ in the $(\varphi, r)$-plane. We further require that the $N_{s}$ $(s \geq 0)$ be chosen such that $\Omega_{0}^{s} \subset \Omega_{0}$.

We note that, by construction, $V_{s}$ and $W_{s}$ are almost constant on each of the sets $I_{s k}$. Fix $\varphi_{s k} \in I_{0} \cap I_{s k}$. For $z=r e^{i \varphi} \in \Omega_{0}^{s k}$, let $f_{s k}(z):=$ 
$\alpha_{s k} \varphi+\beta_{s k}$, where $\alpha_{s k}, \beta_{s k} \in \mathbf{C}$, are chosen such that $f_{s k}\left(e^{i \varphi_{s k}}\right)=$ $V_{s}\left(e^{i \varphi_{s k}}\right)$ and $\partial f_{s k} / \partial \varphi=\alpha_{s k}=W_{s}\left(e^{i \varphi_{s k}}\right)$, so that $\left|\alpha_{s k}\right| \leq 2^{s / 2}$.

Let $f_{s}$ be the function defined on $\Omega_{0}^{s}$ which is equal to $f_{s k}$ on $\Omega_{0}^{s k}$. And let $f=\sum_{s=0}^{\infty} f_{s} \chi_{s}$. Then $f$ is well-defined on some neighbourhood $\Omega_{0}^{\prime}$ of $F_{0}$. It is not too difficult to see that $f$ satisfies (5). In the sequel, we identify $\Omega_{0}$ and $\Omega_{0}^{\prime}$.

Using the localization scheme of Vitushkin (similarly to [4, Lemma 2.2(8), Corollary 6.3]), one can prove that for each $R>0$, there exists $\left\{f_{n}^{R}\right\} \subset L\left(F_{0}^{R}\right)$, where $F_{0}^{R}=F_{0} \cap\{|z| \leq R\}$, such that $f_{n}^{R} \longrightarrow f$ in $C_{\text {jet }}^{1}\left(F_{0}^{R}\right)$ as $n \rightarrow+\infty$ (see [4] and [2, section 2.1]; in our particular case, since the interior of $F_{0}$ is empty and the union of all the lines in $\mathbf{C} \backslash F_{0}$ is everywhere dense, we only need a very simple part of the localization scheme).

Let us now consider the Banach space

$V=\left\{g \in C^{1}\left(\mathbf{R}^{2}\right) \mid\|g\|:=\sup _{z \in \mathbf{R}^{2}}\left\{\max \{|g(z)|,|\nabla g(z)|\}\left(1+|z|^{2}\right)\right\}<\infty\right\}$

with norm $\|\cdot\|$. This space satisfies the conditions (1)-(4) of [2]. From the fact that $V$ is locally equivalent to the space $C^{1}\left(\mathbf{R}^{2}\right)$ and from the approximation properties of $f$ on $F_{0}^{R}$ mentioned above, it follows also that there exists a locally finite family of balls covering $F_{0}$ such that for each ball $B$ in this family and for each $\varepsilon>0$, there exists $g$ such that $L g=0$ on some neighbourhood of $F_{0} \cap \bar{B}$ and $\|f-g\|_{F_{0} \cap \bar{B}}<\varepsilon$ i.e. $f$ is approximable locally on $F_{0}$ in the norm of $V$ by (local) $L$ analytic functions. Theorem 2 in [2] now states that this is equivalent to global approximation, that is, for each $\varepsilon>0$, there exists an $L$-analytic function $g$ on (all of) $F_{0}$ such that $\|f-g\|_{F_{0}}<\varepsilon$.

Denote by $\mathbf{R}_{\infty}^{2}=\mathbf{R}^{2} \cup\{\infty\}$ the one-point compactification of $\mathbf{R}^{2}$. Since $\mathbf{R}_{\infty}^{2} \backslash F_{0}$ is connected and locally connected (that is, $F_{0}$ is a $R K L$-set in the terminology of [2] (the letters stand for Roth-KeldyshLavrentieff)), we can use an analog of Runge's theorem obtained in [2, Theorem 1] to approximate in the norm of $V L$-analytic functions on $F_{0}$ by $L$-entire functions. We thus conclude that we can find an $L$-entire function $h$ such that $\|f-h\|_{F_{0}} \leq 1$. Using the estimate

$$
|\partial \psi(z) / \partial \varphi|<|\nabla \psi(z)||z|,
$$

this gives that (5) is satisfied when $h$ is substituted for $f$.

Now define $v(z)=h(z)$ in $\Omega_{0}$ and $v(z)=U\left(e^{i \arg (z)}\right)$ in $\cup_{j=1}^{\infty} \Omega_{j}$. Then $v \in L(\Omega)$, where $\Omega=\cup_{j=0}^{\infty} \Omega_{j}$ is a neighbourhood of $F$, and $F$ is a $R K L$-set. Thus again by [2, Theorem 1], we can find $u \in L\left(\mathbf{R}^{2}\right)$ with $\|v-u\|_{F} \leq 1$. It suffices to notice, using (6) with $\psi=u-v$, that $u$ is the desired $L$-entire function. Theorem 1 is proved. 
Proof of Theorem 2: Part (a) of Theorem 2 trivially follows from Theorem 1 , since it suffices to extend $g$ from $K$ to $S$ by setting $g=0$ on $S \backslash K$

Suppose that $K \neq S$. The necessity in (b) is also a simple consequence of the proof of Theorem 1. To obtain the sufficiency in (b), we consider the closed set $F=\left\{z=r e^{i \varphi} \in \mathbf{C} \mid e^{i \varphi} \in K, r \geq 1\right\}$ and the function $f(z)=f\left(r e^{i \varphi}\right):=g\left(e^{i \varphi}\right)$ on the $R K L$-set $F$.

An elementary proof (using only well known facts from one-dimensional real analysis) shows that for each $\varepsilon>0$, there exists a finite number of disjoint open $\operatorname{arcs} I_{j}$, whose union $I=\cup I_{j}$ contains $K$, and a function $h_{\varepsilon}$ on $I$ such that $h_{\varepsilon}$ has the form $v_{12}^{*}\left(\right.$ or $v_{1}^{*}$ ) (see Proposition 2) on each $I_{j}$, and

$$
\sup \left\{\left|g\left(e^{i \varphi}\right)-h_{\varepsilon}\left(e^{i \varphi}\right)\right| \mid e^{i \varphi} \in K\right\}<\varepsilon .
$$

Thus $f(z)$ is approximable uniformly on $F$ by functions $h_{\varepsilon}(z)=$ $h_{\varepsilon}\left(e^{i \arg (z)}\right) \in L(F)$.

The end of the proof is now similar to that of Theorem 1. We just need to take the following new approximation space:

$$
V=\left\{\psi \in C\left(\mathbf{R}^{2}\right) \mid\|\psi\|=\sup _{z \in \mathbf{C}}(|\psi(z)|(1+|z|))<\infty\right\} .
$$

Finally, if $K=S$, then $u=u_{g}$ must be bounded in $\mathbf{R}^{2}$, and hence $|\nabla u|$ is also bounded (see the beginning of the proof of Theorem 1). Then, considering $\partial_{1} u$ and $\partial_{2} u$ and using Proposition 1, we reduce the proof to an application of Liouville's Theorem for holomorphic functions.

\section{References}

1. A. V. Bitsadze, "Boundary-value problems for second order elliptic equations," North-Holland Series in Applied Mathematics and Mechanics 5, North-Holland, Amsterdam, 1968.

2. A. Boivin And P. V. Paramonov, Approximation by meromorphic and entire solutions of elliptic equations in Banach spaces of distributions, Sb. Math. 189(4) (1998), 481-502.

3. D. GAIER, "Lectures on Complex Approximation," Birkhäuser, Boston Basel Stuttgart, 1987.

4. P. V. Paramonov, On harmonic approximation in the $C^{1}$-norm, Math. USSR-Sb. 71(1) (1992), 183-207.

5. P. V. Paramonov and K. Yu. Fedorovski, On $C^{1}$-approximation of functions by polynomial solutions of homogeneous elliptic 
equations of second order on compact sets in $\mathbf{R}^{2}$, Dep. in VINITI 2965-B96 (1996), 1-15. (In Russian).

6. P. V. Paramonov and J. Verdera, Approximation by solutions of elliptic equations on closed subsets of Euclidean space, Math. Scand. 74 (1994), 249-259.

7. A. Rотн, Approximationseigenschaften und strahlengrenzwerte meromorpher und ganzer funktionen, Comment. Math. Helv. 11 (1938), 77-125.

André Boivin:

Department of Mathematics

University of Western Ontario

London (Ontario)

CANADA N6A 5B7

e-mail: boivin@uwo.ca
Peter V. Paramonov:

Mechanics and Mathematics Faculty

Moscow State (Lomonosov) University

119899 Moscow

RUSSIA

e-mail: petr@paramonov.msk.ru

Primera versió rebuda el 10 de març de 1998,

darrera versió rebuda el 23 de juny de 1998 